\title{
Transversal and cotransversal matroids via their representations.
}

\author{
Federico Ardila* \\ Submitted: May 23, 2006; Accepted: Feb 27, 2007; Published: Mar 5, 2007 \\ Mathematics Subject Classification: 05B35; 05C38; 05A99
}

\begin{abstract}
.
It is known that the duals of transversal matroids are precisely the strict gammoids. We show that, by representing these two families of matroids geometrically, one obtains a simple proof of their duality.
\end{abstract}

\section{0}

This note gives a new proof of the theorem, due to Ingleton and Piff [3], that the duals of transversal matroids are precisely the strict gammoids. Section 1 defines the relevant objects. Section 2 presents explicit representations of the families of transversal matroids and strict gammoids. Section 3 uses these representations to prove the duality of these two families.

\section{1}

Matroids and duality. A matroid $M=(E, \mathcal{B})$ is a finite set $E$, together with a non-empty collection $\mathcal{B}$ of subsets of $E$, called the bases of $M$, which satisfy the following axiom: If $B_{1}, B_{2}$ are bases and $e$ is in $B_{1}-B_{2}$, there exists $f$ in $B_{2}-B_{1}$ such that $\left(B_{1}-e\right) \cup f$ is a basis.

If $M=(E, \mathcal{B})$ is a matroid, then $\mathcal{B}^{*}=\{E-B \mid B \in \mathcal{B}\}$ is also the collection of bases of a matroid $M^{*}=\left(E, \mathcal{B}^{*}\right)$, called the dual of $M$.

Representable matroids. Matroids can be thought of as providing a combinatorial abstraction of linear independence. If $V$ is a set of vectors in a vector space and $\mathcal{B}$ is the

\footnotetext{
${ }^{*}$ federico@math.sfsu.edu. Dept. of Mathematics, San Francisco State University, San Francisco, CA, USA. Supported by NSF grant DMS-9983797.
} 
collection of maximal linearly independent subsets of $V$, then $M=(V, \mathcal{B})$ is a matroid. Such a matroid is called representable, and $V$ is called a representation of $M$.

Transversal matroids. Let $A_{1}, \ldots, A_{r}$ be subsets of $[n]=\{1, \ldots, n\}$. A transversal (or system of distinct representatives $)$ of $\left(A_{1}, \ldots, A_{r}\right)$ is an $r$-subset of $[n]$ whose elements can be labelled $\left\{e_{1}, \ldots, e_{r}\right\}$ in such a way that $e_{i}$ is in $A_{i}$ for each $i$. The transversals of $\left(A_{1}, \ldots, A_{r}\right)$ are the bases of a matroid on $[n]$.

Such a matroid is called a transversal matroid, and $\left(A_{1}, \ldots, A_{r}\right)$ is called a presentation of the matroid. This presentation can be encoded in the bipartite graph $H$ with "top" vertex set $T=[n]$, "bottom" vertex set $B=\{\widehat{1}, \ldots, \widehat{r}\}$, and an edge joining $j$ and $\widehat{i}$ whenever $j$ is in $A_{i}$. The transversals are the $r$-sets in $T$ that can be matched to $B$. We will denote this transversal matroid by $M[H]$.

Strict gammoids. Let $G$ be a directed graph with vertex set $[n]$, and let $A=\left\{v_{1}, \ldots, v_{r}\right\}$ be a subset of $[n]$. We say that an $r$-subset $B$ of $[n]$ can be linked to $A$ if there exist $r$ vertex-disjoint directed paths, each of which has its initial vertex in $B$ and its final vertex in $A$. Each individual path is allowed to have repeated vertices and edges. Such a collection of $r$ paths is called a routing from $B$ to $A$. The $r$-subsets which can be linked to $A$ are the bases of a matroid denoted $L(G, A)$. We can assume that the vertices in $A$ are sinks of $G$; that is, that there are no edges coming out of them. This is because the removal of those edges does not affect the matroid $L(G, A)$.

The matroids that arise in this way are called strict gammoids or cotransversal matroids. The purpose of this note is to give a new proof of the following theorem, due to Ingleton and Piff.

Theorem 1. [3] Strict gammoids are precisely the duals of transversal matroids.

\section{2}

Let $\mathbb{K}$ be the field of fractions of the ring of formal power series in the indeterminates $x_{i j}$ indexed by $1 \leq i \leq r$ and $1 \leq j \leq n$. We now show how transversal matroids and strict gammoids can be represented over $\mathbb{K}^{1}$

A representation of transversal matroids. Let $M$ be a transversal matroid on the set $[n]$ with presentation $\left(A_{1}, \ldots, A_{r}\right)$. Let $X$ be the $r \times n$ matrix whose $(i, j)$ entry is $-x_{i j}$ if $j \in A_{i}$ and is 0 otherwise. The columns of $X$ are a representation of $M$ in the vector space $\mathbb{K}^{r}$. To see this, consider the columns $j_{1}, \ldots, j_{r}$. They are independent when their determinant is non-zero, and this happens as soon as one of the $r$ ! summands of the determinant is non-zero. But $\pm X_{\sigma_{1} j_{1}} \cdots X_{\sigma_{r} j_{r}}$ (where $\sigma$ is a permutation of $[r]$ ) is nonzero if and only if $j_{1} \in A_{\sigma_{1}}, \ldots, j_{r} \in A_{\sigma_{r}}$. So the determinant is non-zero if and only if $\left\{j_{1}, \ldots, j_{r}\right\}$ is a transversal.

We will find it convenient to choose a transversal $j_{1} \in A_{1}, \ldots, j_{r} \in A_{r}$ at the outset, and normalize the rows to have the $\left(i, j_{i}\right)$ entry be $-x_{i j_{i}}=1$ for $1 \leq i \leq r$.

\footnotetext{
${ }^{1}$ It is possible to carry out the same constructions over $\mathbb{R}$, but special care is required to handle the issue of convergence of the infinite sums that will arise.
} 
Example 1. Let $n=6$ and $A_{1}=\{1,2,3\}, A_{2}=\{2,4,5\}, A_{3}=\{3,5,6\}$. The corresponding bipartite graph $H$ is shown below.

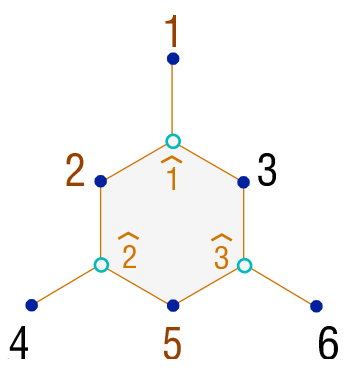

If we choose the transversal $1 \in A_{1}, 2 \in A_{2}, 3 \in A_{3}$, we obtain a representation for the transversal matroid $M[H]$, given by the columns of the following matrix:

$$
X=\left(\begin{array}{cccccc}
1 & -a & -b & 0 & 0 & 0 \\
0 & 1 & 0 & -c & -d & 0 \\
0 & 0 & 1 & 0 & -e & -f
\end{array}\right)
$$

A representation of strict gammoids. Let $M=L(G, A)$ be a strict gammoid. Say $G$ has vertex set $[n]$, and assume $A=\{r+1, \ldots, n\}$. Any edge $i \rightarrow j$ of $G$ has $i \leq r$, so we can assign to it weight $x_{i j}$. Let the weight of a path in $G$ be the product of the weights on its edges. For each vertex $i$ of $G$ and each sink $a$ in $A$, let $p_{i a}$ be the sum of the weights of all paths in $G$ which start at vertex $i$ and end at sink $a$. We allow paths with repeated vertices and edges in this sum, so there may be infinitely many such paths; however, the number of paths of a given weight is finite, so $p_{i a}$ is a well-defined element of $\mathbb{K}^{2}$

Let $Y$ be the $(n-r) \times n$ matrix whose $(a, i)$ entry is $p_{i a}$. The columns of $Y$ are a representation of $M$. To see this, recall the Lindström-Gessel-Viennot theorem, which states that the determinant of the submatrix with columns $i_{1}, \ldots, i_{n-r}$ is equal to the signed sum of the weights of the routings from $\left\{i_{1}, \ldots, i_{n-r}\right\}$ to $A .^{3}$

Notice that two routings $R_{1}$ and $R_{2}$ having the same weight must have the same multiset of edges. They can only differ in the order in which the $k$ th path of $R_{1}$ and the $k$ th path of $R_{2}$ traverse the same multiset of edges, as they go from $i_{k}$ to $j_{k}$. But then $R_{1}$ and $R_{2}$ will also have the same sign. We conclude that there is no cancellation in the signed sum under consideration. Therefore the determinant of the submatrix with columns $i_{1}, \ldots, i_{n-r}$ is non-zero if and only if the signed sum is non-empty; that is, if and only if $\left\{i_{1}, \ldots, i_{n-r}\right\}$ can be linked to $A$.

Example 2. Consider the directed graph $G$ shown below, where all edges point down, and the set $A=\{4,5,6\}$.

\footnotetext{
${ }^{2}$ In fact, $p_{i a}$ is a rational function in the $x_{i j}$ s. For a proof, see [10, Theorem 4.7.2].

${ }^{3}$ If the $k$ th path in a routing starts at $i_{k}$ and ends at $j_{k}$, then the sign of the routing is the sign of the permutation $j_{1} \ldots j_{n-r}$ of $A$.
} 


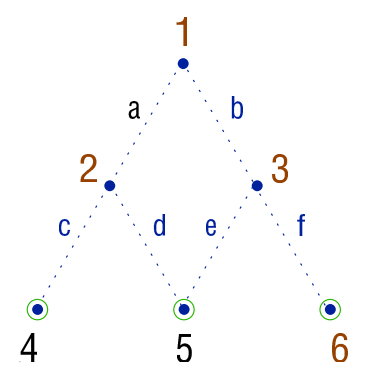

The representation we obtain for the strict gammoid $L(G, A)$ is given by the columns of the following matrix:

$$
Y=\left(\begin{array}{cccccc}
a c & c & 0 & 1 & 0 & 0 \\
a d+b e & d & e & 0 & 1 & 0 \\
b f & 0 & f & 0 & 0 & 1
\end{array}\right)
$$

Representations of dual matroids. If a rank- $r$ matroid $M$ is represented by the columns of an $r \times n$ matrix $A$, we can think of $M$ as being represented by the $r$-dimensional subspace $V=\operatorname{rowspace}(A)$ in $\mathbb{K}^{n}$. The reason is that, if we consider any other $r \times n$ matrix $A^{\prime}$ with $V=\operatorname{rowspace}\left(A^{\prime}\right)$, the columns of $A^{\prime}$ also represent $M$.

This point of view is very amenable to matroid duality. If $M$ is represented by the $r$-dimensional subspace $V$ of $\mathbb{K}^{n}$, then the dual matroid $M^{*}$ is represented by the $(n-r)$ dimensional orthogonal complement $V^{\perp}$ of $\mathbb{K}^{n}$.

Notice that the rowspaces of the matrices $X$ and $Y$ in the examples above are orthogonally complementary. That is, essentially, the punchline of this story.

\section{3}

Directed graphs with sinks and bipartite graphs with complete matchings. Given a directed graph $G$ and a subset $A$ of its set of sinks, we construct an undirected graph $H$ as follows. We first split each vertex $v$ not in $A$ into a "top, incoming" vertex $v$ and a "bottom, outgoing" vertex $\widehat{v}$, and draw an edge between them. Then we replace each edge $u \rightarrow v$ of $G$ with an edge between the outgoing $\widehat{u}$ and the incoming $v$.

More concretely, given a directed graph $G$ with vertex set $V$, and given a set $A$ of sinks of $G$, we construct a bipartite graph $H$, together with a fixed bipartition and a fixed complete matching. The top vertex set in the bipartition is $V$, and the bottom vertex set is a copy $\widehat{V}-\widehat{A}$ of $V-A$. The complete matching is obtained by joining the top $u$ and the bottom $\widehat{u}$ for each $u$ in $V-A$. Then, for $u \neq v$, we join the bottom $\widehat{u}$ and the top $v$ in $H$ if and only if $u \rightarrow v$ is an edge of $G$.

Conversely, if we are given the bipartite graph $H$, a bipartition of $H^{4}$ and a complete matching of $H$, it is clear how to recover $G$ and $A$. The resulting $G$ and $A$ will depend on which bipartition and matching are used. Observe that if we start with the directed graph $G$ and sinks $A$ of Example 2, we obtain the bipartite graph $H$ of Example 1 .

\footnotetext{
${ }^{4}$ which is unique if $H$ is connected
} 
Having laid the necessary groundwork, we can now present our proof of Theorem 1.

Proof of Theorem 1: Duality of transversal matroids and strict gammoids. We constructed a correspondence between a directed graph $G$ with a specified subset $A$ of its set of sinks, and a bipartite graph $H$ with a specified bipartition and a specified complete matching. Now we show that, in this correspondence, the strict gammoid $L(G, A)$ is dual to the transversal matroid $M[H]$. We have constructed a subspace of $\mathbb{K}^{n}$ representing each one of them. By the remarks at the end of Section 2, it suffices to show that these two subspaces are orthogonally complementary, as observed in Examples 1 and 2.

Our representation of $M[H]$ is given by the columns of the $r \times n$ matrix $X$ whose $(i, i)$ entry is 1 , and whose $(i, j)$ entry, for $i \neq j$, is $-x_{i j}$ if $i \rightarrow j$ is an edge of $G$ and is 0 otherwise. Think of the $x_{i j} \mathrm{~s}$ as weights on the edges of $G$. For a vector $y \in \mathbb{K}^{n}$, the $i$ th entry of the column vector $X y$ is $y_{i}-\sum_{j \in N(i)} x_{i j} y_{j}$, where the sum is over the set $N(i)$ of vertices $j$ such that $i \rightarrow j$ is an edge of $G$. It follows that $y$ is in the nullspace of $X$ when, for each vertex $i$ of $G$,

$$
y_{i}=\sum_{j \in N(i)} x_{i j} y_{j}
$$

As before, let $p_{i a}$ be the sum of the weights of the paths from $i$ to $a$ in $G$. Now we observe that

$$
p_{i a}=\sum_{j \in N(i)} x_{i j} p_{j a}
$$

To see this, notice that the left hand side enumerates all paths from $i$ to $a$, and the right hand side enumerates the same paths by grouping them according to the first vertex $j$ that they visit after $i$. Therefore $\left(p_{1 a}, \ldots, p_{n a}\right)$, the ath row of our representation $Y$ of $L(G, A)$, is in the nullspace of $X$. Since each row of $Y$ is in the nullspace of $X$, $\operatorname{rowspace}(Y) \subseteq \operatorname{nullspace}(X)$. But

$$
\begin{aligned}
\operatorname{dim}(\operatorname{rowspace}(Y)) & =\operatorname{rank}(L(G, A))=n-r, \text { and } \\
\operatorname{dim}(\operatorname{nullspace}(X)) & =n-\operatorname{dim}(\operatorname{rowspace}(X))=n-\operatorname{rank}(M[H])=n-r,
\end{aligned}
$$

so in fact these two subspaces are equal. It follows that $\operatorname{rowspace}(X)$ and $\operatorname{rowspace}(Y)$ are orthogonal complements. This completes our proof of Theorem 1.

\section{4}

For more information on matroid theory, Oxley's book [8] is a wonderful place to start. The representation of transversal matroids shown here is due to Mirsky and Perfect [7]. The representation of strict gammoids that we use was constructed by Mason [6] and further explained by Lindström. ${ }^{5}[5]$

This note is a small side project of [1]. While studying the combinatorics of generic flag arrangements and its implications on the Schubert calculus, we became interested in

\footnotetext{
${ }^{5}$ It is in this context that he discovered what is now commonly known as the Lindström-Gessel-Viennot theorem [2]. This theorem was also discovered and used earlier by Karlin and MacGregor [4].
} 
the strict gammoid of Example 2 and its representations; we proved that it is the matroid of the arrangement of lines determined by intersecting three generic complete flags in $\mathbb{C}^{3}$. Similarly, the analogous strict gammoid in a triangular array of size $n$ is the matroid of the line arrangement determined by three generic flags in $\mathbb{C}^{n}$.

I would like to thank Sara Billey for several helpful discussions, and Laci Lovász and Jim Oxley for help with the references. I also thank the referee for several suggestions which improved the presentation.

\section{References}

[1] F. Ardila and S. Billey. Flag arrangements and triangulations of products of simplices. Preprint, math. C0/0605598, 2006.

[2] I. Gessel and X. Viennot. Binomial determinants, paths and hook formulae. Adv. Math 58 (1985) 300-321.

[3] A. Ingleton and M. Piff. Gammoids and transversal matroids. J. Combinatorial Theory Ser. B 15 (1973) 51-68.

[4] S. Karlin and G. MacGregor. Coincidence probabilities. Pacific J. Math. 9 (1959) 1141-1164.

[5] B. Lindström. On the vector representations of induced matroids. Bull. London Math. Soc. 5 (1973) $85-90$.

[6] J. Mason. On a class of matroids arising from paths in graphs. Proc. London Math. Soc. (3) 25 (1972) 55-74.

[7] L. Mirsky and H. Perfect. Applications of the notion of independence to problems of combinatorial analysis. J. Combinatorial Theory 2 (1967) 327-357.

[8] J. G. Oxley. Matroid theory. Oxford University Press. New York, 1992.

[9] M. J. Piff and D. J. A. Welsh. On the vector representation of matroids. J. London Math. Soc. (2) 2 284-288.

[10] R. P. Stanley. Enumerative combinatorics, vol. 1. Cambridge University Press. Cambridge, 1997. 